\title{
Platelet-rich plasma effects on degenerative disc disease: analysis of histology and imaging in an animal model
}

Authors Gregory B Gullung ${ }^{1}$, James W Woodall ${ }^{1}$, Michelle A Tucci ${ }^{1}$, Judy James ${ }^{2}$, David A Black ${ }^{1}$, Robert A McGuire ${ }^{1}$

Institutions ${ }^{1}$ Department of Orthopaedics, University of Mississippi Medical Center, Jackson, MS, USA

${ }^{2}$ Department of Radiology, University of Mississippi Medical Center, Jackson, MS, USA

\section{ABSTRACT}

Study design: Randomized controlled animal trial.

Objectives: To analyze the early and late phase effects of platelet-rich plasma (PRP) injection into and around the damaged intervertebral disc using an animal model, and to evaluate the needle puncture technique in creating a degenerative disc model.

Methods: The L4-L5 intervertebral disc of 18 adult Sprague-Dawley rats was injured with a 21-gauge needle. Animals received an immediate injection of PRP, or a delayed injection of PRP 2 weeks postinjury, or no further intervention (sham) ( $\mathrm{n}=6 /$ group). Six uninjured controls were used. Magnetic resonance imaging (MRI) was performed for a control at time 0 and each group at 4 weeks postinjury, this allowed resolution of inflammation and an accurate assessment of healing. Specimens were collected from immediate PRP group at 2 and 4 weeks postinjury, and the delayed PRP group at 4 and 6 weeks postinjury. The sham and control specimens were collected at 2, 4, and 6 weeks postinjury. Each disc was sectioned and analyzed subjectively for overall structure, cellularity, and water content (utilizing MRI). Disc height was measured using descriptive statistics and one-way analysis of variance (ANOVA) with Tukey post hoc analysis. It was presumed both groups injected with PRP would have better outcomes than the sham group.

Results: The sham group had clear degenerative changes with loss of organizational structure, empty space, fibrous tissue, and inflammatory cells, indicating needle puncture leads to degeneration. The PRP treated groups had fibers that were damaged with empty spaces and inflammatory cells. However, there was maintenance of the ring structure and the nucleus appeared to have a healthy central portion. Overall, both PRP-treated groups retained more normal morphologic features, contained fewer

The author has no conflict of interest for this study. No funding was received for research. 
inflammatory cells, and had higher fluid content on MRI; however the effect was more pronounced in the immediate injection group. The disc height was significantly different in the sham and immediate injection group at the 4-week interval.

Conclusions: This percutaneous needle puncture technique is an effective method for creating a degenerative disc model without posterior destabilization of the spine. The administration of PRP has a protective effect on damaged discs in the acute and delayed injection settings representing clinical treatment with PRP in the early versus late stages of the degenerative process. It appears that earlier intervention in the disease process would be more beneficial than PRP treatment of already severely degenerated discs.

\section{STUDY RATIONALE AND CONTEXT}

The current conservative and surgical modalities of treating degenerative disc disease are aimed at relief of symptoms. Evidence supports the use of platelet-rich plasma (PRP) for various tendinopathies by decreasing inflammation and fibrosis $[1,2]$. The potential benefit of PRP injection in treating or preventing progression of degenerative disc disease has not been critically evaluated in vivo.

\section{OBJECTIVES}

To analyze the early and late phase effects of PRP injection into and around the damaged intervertebral disc using an animal model, and to evaluate the needle puncture technique in creating a degenerative disc model.

\section{METHODS}

Study design: Randomized animal study.

- Institutional Review Board and Institutional Animal Care and Use Committee (IACUC) approval obtained.

- Twenty-four male Sprague Dawley rats were included (Table 1). Exclusion criteria were apparent dysfunction or injury to hindlimbs or lumbosacral spine, as well as postoperative neurovascular injury. No animals were excluded (Fig $\mathbf{1}$ ).

- Animals were anesthetized with $7.5 \mathrm{mg} / \mathrm{kg}$ ketamine and $10 \mathrm{mg} / \mathrm{kg}$ xylazine.
- A single surgeon then injured the L4-L5 intervertebral disc in 18 animals, from a left-sided percutaneous approach under image intensifier, using a 21-gauge needle [3] (Fig 2). Six were kept as uninjured controls.

- The 18 injured animals were then randomly assigned to one of three groups: no further intervention (sham) $(\mathrm{n}=6)$; immediate injection $(\mathrm{n}=6)$ of $0.1 c c$ PRP in and around the disc; or a delayed injection $(n=6)$ of 0.1cc PRP 2 weeks after injury, to allow for disc degeneration.

- Postoperatively they were given $5 \mathrm{mg} / \mathrm{kg}$ carprofen for 4 days for pain control.

\section{Outcomes}

- Clinically, specimens were observed on a daily basis for injection-site healing, normal use of hindlimbs and tail, as well as normal feeding and grooming behavior.

- T2 magnetic resonance imaging (MRI) was obtained to evaluate disc hydration on the control at 0,2 , and 4 weeks and in the immediate and sham group at 2 and 4 weeks after injury, and the delayed group at 4 weeks after injury (2 weeks after injection). At 4 weeks after injury the inflammation has resolved sufficiently to allow for accurate assessment of healing.

- Three specimens were collected from each of the PRP groups at 2 and 4 weeks after PRP injection, two specimens were collected from sham and control groups at 2, 4, and 6 weeks after injury.

- Injured spinal segments were cleaned of soft tissue and decalcified in 2.4 $\mathrm{M}$ formic acid, placed in paraffin, sliced at $5 \mu \mathrm{m}$, hematoxylin-eosin stained, and photographed under $4 \mathrm{X}$ magnification.

- NIH Image $J$ software measured the average disc height after placement on a standardized grid. Disc hydration was subjectively evaluated as good, fair, or poor based on the uninjured control. Architecture was based in integrity of annulus and nucleus pulposus, as well as end-plate cartilage, and presence of inflammatory cells.

\section{Analysis}

- A reviewer blinded to intervention qualitatively evaluated the integrity of the annulus fibrosis, nucleus pulposus, cartilage end plate, and cellularity, while a different blinded reviewer evaluated MRI findings. Although blinded reviewers were used, their analysis was not subject to verification, and personal bias may have influenced the results.

- Disc height was evaluated using descriptive statistics; one-way analysis of variance with Tukey multiple comparisons procedure. 
Table 1 Specimen characteristics.

\begin{tabular}{|c|c|c|c|c|c|c|}
\hline \multirow{2}{*}{$\begin{array}{l}\text { Specimen characteristics } \\
\text { Gender }\end{array}$} & \multirow{2}{*}{$\begin{array}{l}\text { Control } \\
(n=6)\end{array}$} & \multicolumn{2}{|c|}{$\begin{array}{l}\text { Immediate injection of PRP* } \\
(n=6)\end{array}$} & \multicolumn{2}{|c|}{$\begin{array}{l}\text { Delayed injection of PRP } \\
(n=6)\end{array}$} & \multirow{2}{*}{$\begin{array}{l}\text { Sham PRP injection } \\
(n=6) \\
M\end{array}$} \\
\hline & & M & M & M & M & \\
\hline Time to harvest ${ }^{* *}$ & N/A & 2 wk $(n=3)$ & 4 wk $(n=3)$ & 2 wk $(n=3)$ & 4 wk $(n=3)$ & N/A \\
\hline Weight, $g( \pm S D)$ & $500.5( \pm 17.5)$ & $490.5( \pm 13.7)$ & $466.6( \pm 31.0)$ & $502.3( \pm 12.0)$ & $498.6( \pm 21.1)$ & $501.2( \pm 21.5)$ \\
\hline Level injured & $\mathrm{N} / \mathrm{A}$ & L4-L5 & L4-L5 & L4-L5 & L4-L5 & L4-L5 \\
\hline Time of PRP injection, wk & $N / A$ & 0 & 0 & 2 & 2 & $\mathrm{~N} / \mathrm{A}$ \\
\hline
\end{tabular}

* PRP indicates platelet-rich plasma; N/A, not applicable.

** weeks post PRP injection

Fig 1 Patient sampling and selection.
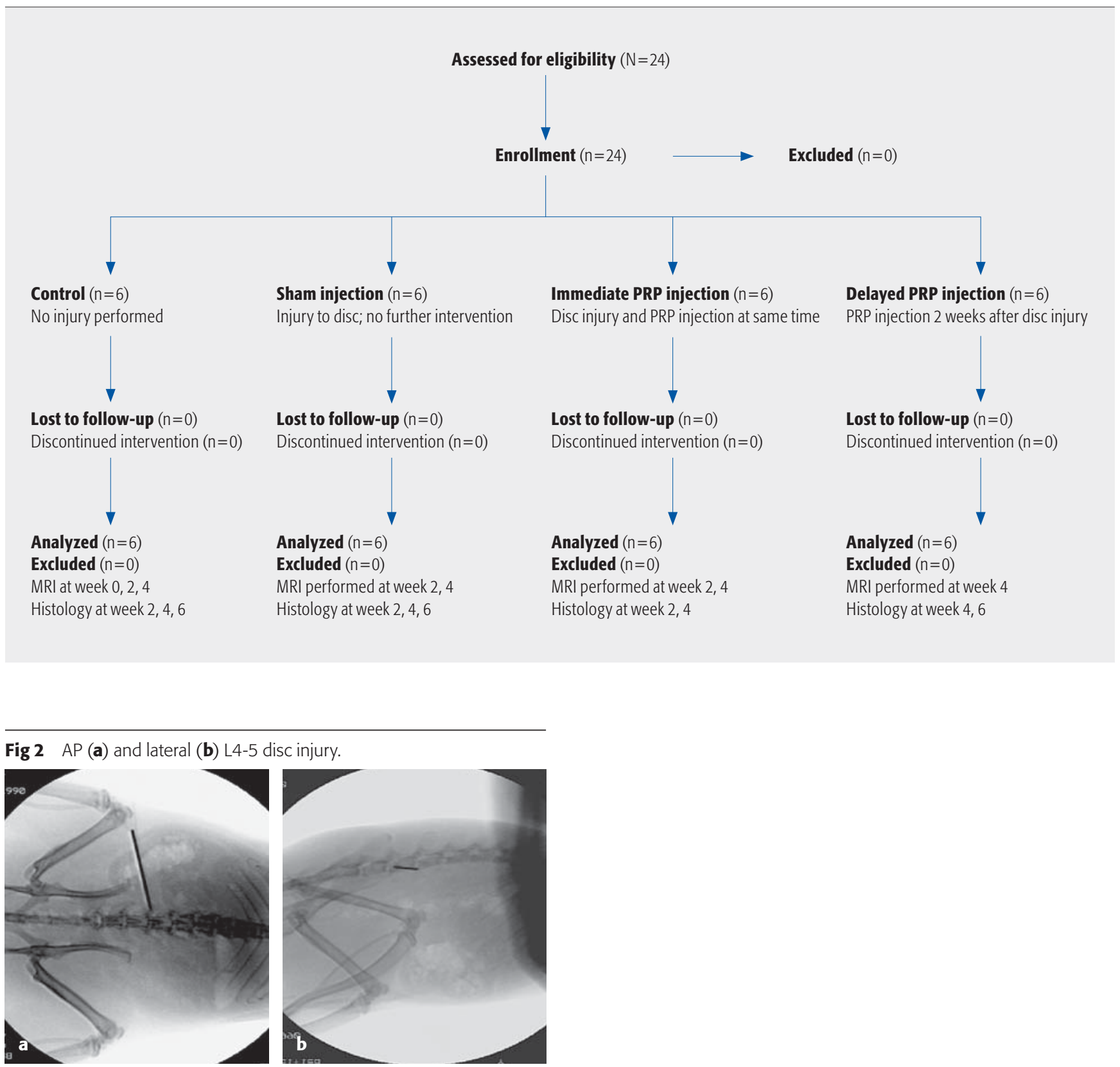


\section{RESULTS}

- Clinically, no specimens were excluded for neurovascular injury or failure to thrive.

- Eight rats held the left hind leg flexed immediately after surgery, but resumed normal activity within 2 days. The transient nature of this finding indicates a local inflammatory origin rather than damage to the nervous structures.

- MRI evaluation led to qualitative evaluation of disc hydration as good, fair, or poor (Fig 3) with the following ratings:

- Control discs: good-white appearance

- Immediate and delayed PRP injection groups: fair-grey appearance

- Sham group: poor- black appearance
- Histological analysis showed distinct differences among the groups (Figs $\mathbf{4}$ and $\mathbf{5}$ ).

- The sham group had clear degenerative changes with loss of structure, empty spaces, fibrous tissue, and large inflammatory cells.

- Immediate injection PRP group had fibers that were damaged with some empty spaces and inflammatory cells. However, structures of the annulus and nucleus pulposus were largely preserved.

- Delayed injection PRP group showed more damage and inflammatory cells than the immediate injection group, but still retained easily identifiable annulus and nucleus structures.

- Assessment of disc height (Table 2) revealed a significant difference between the sham and immediate injection groups at 4 weeks after PRP injection after evaluation with ANOVA and Tukey post hoc analysis ( $P=.032$ and $P=.045$, respectively) (Fig 6). Evaluation was performed for all groups at 4 weeks after injury, and for control, sham, and delayed PRP injection groups at 6 weeks after injury.

Fig 3 Magnetic resonance imaging L4-5 disc (line); control (a), sham (b), immediate injection 2 weeks post PRP (c), immediate injection 4 weeks post PRP (d), delayed injection 2 weeks post PRP.
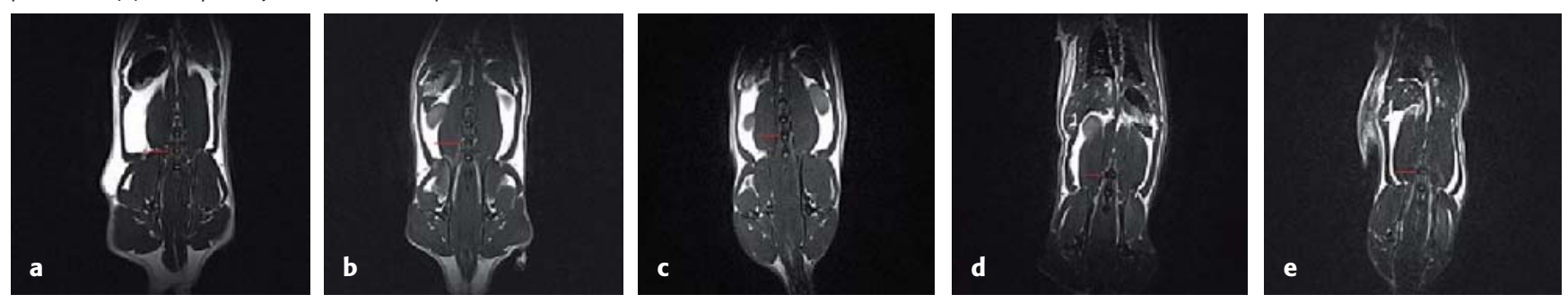

Fig 4 Control (a), sham (b), immediate platelet-rich plasma injection (c), and delayed platelet-rich plasma injection (d).
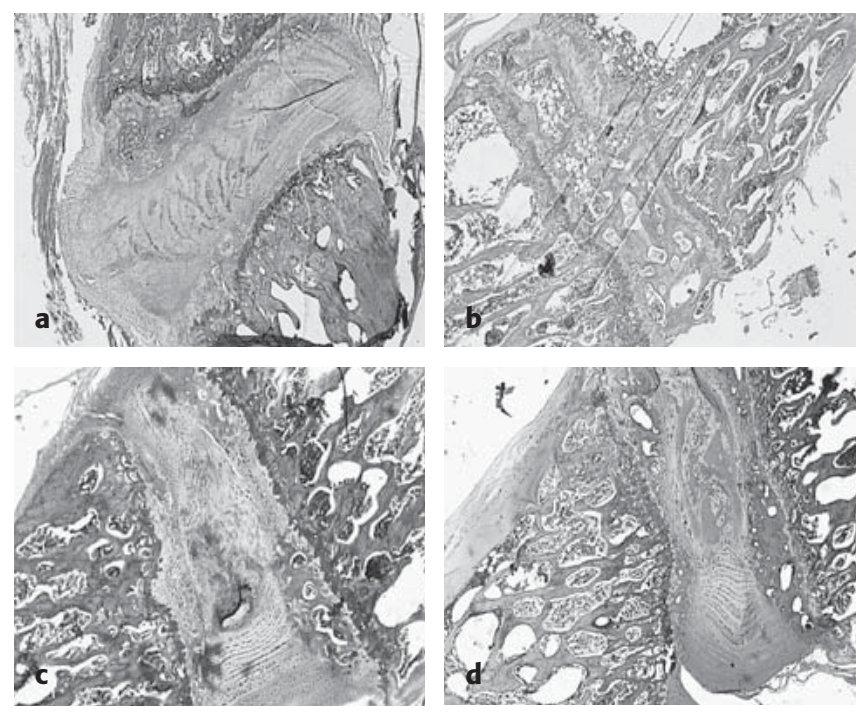

Fig 5 a-c Six-week postinjury histology, control (a), sham (b), and delayed platelet-rich plasma injection (c).
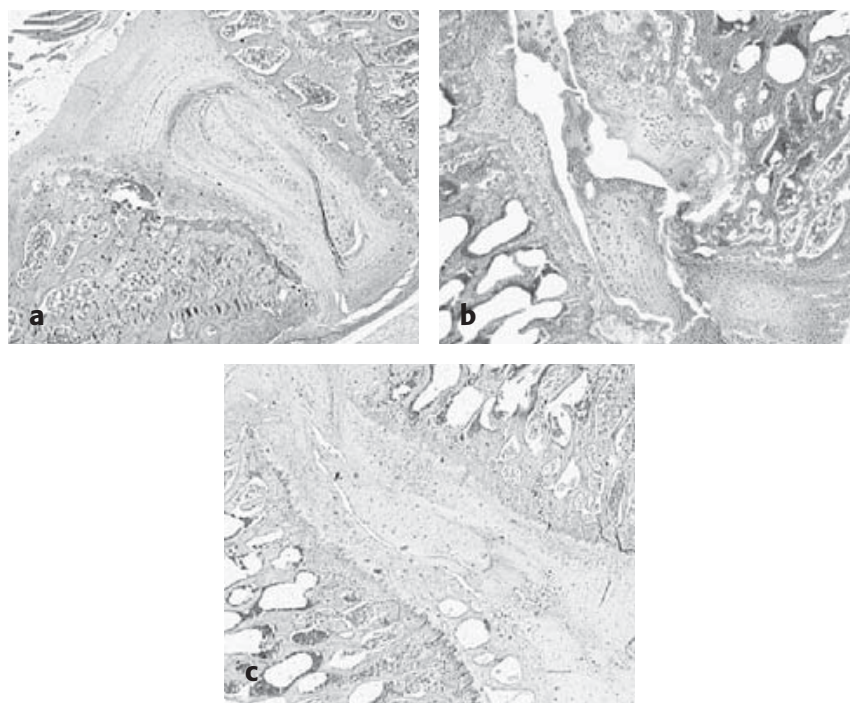


\section{DISCUSSION}

- PRP contains growth factors such as IGF-1, PDGF, EGF, and TGF- $\beta$ [4], which have been shown to stimulate cell proliferation, proteoglycan synthesis, and glycosaminoglycan synthesis in vitro [5, 6], leading to an increase in disc height [7]; as well as decrease the number of apoptotic cells in injured discs in animal models $[8,9]$.

- The sustained release of isolated growth factor in this animal model showed the ability of IGF- 1 and TGF- $\beta$ to preserve degenerating discs [10]. However, the cells were histologically abnormal displaying hypertrophic character. These findings suggest an imbalance in the factor effect when delivered in an isolated manner. In contrast, the current study yielded disc cells of normal histological character. In this study PRP was delivered which contains these growth factors in concentrate but in physiological proportions.

Table 2 Disc height measurement at 4 and 6 weeks after injury.

\begin{tabular}{lll}
\hline Group & $\mathbf{4}$ wk, mean & $\mathbf{6 ~ w k}$, mean \\
\hline Control $( \pm S D), m m$ & $0.768( \pm 0.167)$ & $1.374( \pm 0.345)$ \\
\hline Sham $( \pm S D), m m$ & $0.500( \pm 0.052)$ & $1.362( \pm 0.403)$ \\
\hline $\begin{array}{l}\text { Immediate PRP injection } \\
( \pm S D), m m\end{array}$ & $0.929( \pm 0.327)$ & $\mathrm{N} / \mathrm{A}^{*}$ \\
\hline $\begin{array}{l}\text { Delayed PRP injection } \\
( \pm S D), m m\end{array}$ & $0.612( \pm 0.114)$ & $1.173( \pm 0.374)$ \\
\hline
\end{tabular}

* N/A indicates "not applicable". Last specimens harvested at 4 weeks after injection of platelet-rich plasma, so no immediate injection group existed at 6 weeks after injury.

Fig 6 Asterisk indicates significant difference; statistics grouped the 2-week after injection/4-week after injury (delayed platelet-rich plasma [PRP] injection group) with the 4-week after injection/4-week after injury.

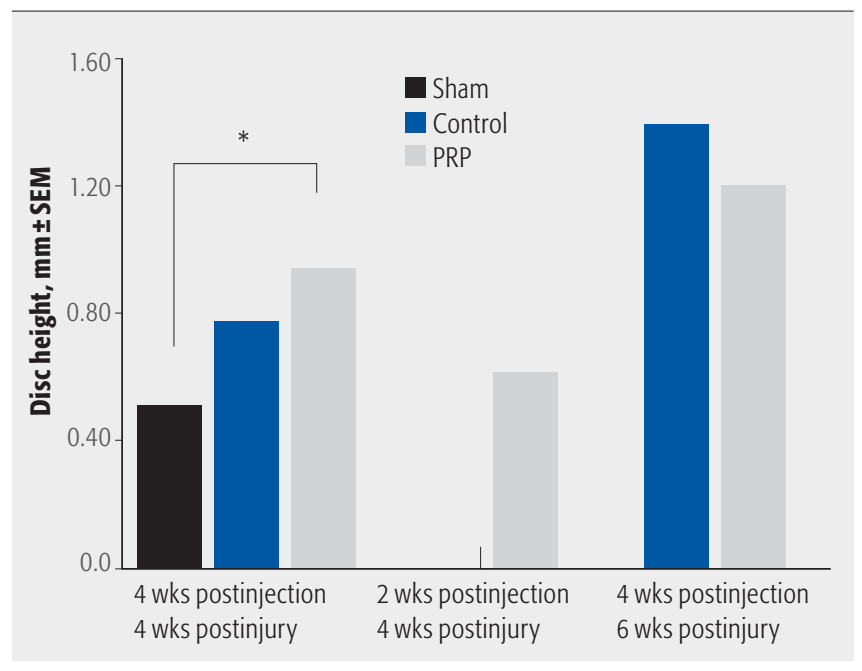

- This compares with our study in that the PRP-treated discs trended toward increased height and definitely had preservation of architecture and cellularity compared with the injured group with no PRP treatment $[5,7]$. However, the previous studies were in vitro or used a microsphere delivery system where direct injection into a living animal model was utilized in this study.

- The techniques used in this randomized controlled trial allowed us to injure and evaluate the disc with minimal undue discomfort to the specimens [3]. Moreover, no specimens were excluded for any reason.

- We recognize that animals do not have vertical loading of the spine; however, the needle puncture technique was effective in creating degenerative discs compared with uninjured controls. Given this, the puncture technique would likely produce a degenerative disc in an upright model, but the effect of PRP may not be as noticeable given the increased forces on the discs.

- Surprisingly, even after the disc was damaged and allowed to degenerate the application of PRP prevented further degeneration when evaluated histologically and by fluid content on MRI, and may have had some regeneration of disc tissue [5, 7]. However, disc height did not seem to be significantly affected.

- The immediate PRP-treated discs had better maintenance of structure, cellularity, hydration, and had a significant difference in disc height from the untreated injured group. It appears early intervention with PRP is protective of degenerating intervertebral discs.

\section{CONCLUSIONS}

This study provides further evidence, in animal models, that the use of an individual's own healing factors in the form of PRP can provide protection for and possibly regeneration of damaged intervertebral discs.

This image-guided and muscle-sparing needle puncture technique provides a novel minimally invasive reliable degenerative disc model. This model achieves disc degeneration without muscle stripping and destabilization of the spine. 


\section{REFERENCES}

1. Gaweda K, Tarczynska M, Krzyzanowski W (2010) Treatment of Achilles tendinopathy with platelet-rich plasma. Int J Sports Med; 31(8):577-583.

2. Gosens T, Peerbooms JC, van Laar W, et al (2011) Ongoing positive effect of plateletrich plasma versus corticosteroid injection in lateral epicondylitis: a double-blind randomized controlled trial with 2-year follow-up. Am J Sports Med; 39(6):1200-1208.

3. Elliott DM, Yerramalli CS, Beckstein JC, et al (2008) The effect of relative needle diameter in puncture and sham injection animal models of degeneration. Spine (Phila Pa 1976); 33(6):588-596.

4. El-Sharkawy H, Kantarci A, Deady J, et al (2007) Platelet-rich plasma: growth factors and pro- and anti-inflammatory properties. J Periodontal; 78(4):661-669.

5. Akeda K, An HS, Pichika R, et al (2006) Platelet-rich plasma (PRP) stimulates the extracellular matrix metabolism of porcine nucleus pulposus and anulus fibrosis cells cultured in alginate beads. Spine (Phila Pa 1976); 33(9):959-966.

6. Chen WH, Lo WC, Lee JJ, et al (2006) Tissue-engineered intervertebral disc and chondrogenesis using human nucleus pulposus regulated through TGF-betal in platelet-rich plasma. J Cell Physiol; 209(3):744-754.

7. Chen WH, Liu HY, Lo WC, et al (2009) Intervertebral disc regeneration in an ex vivo culture system using mesenchymal stem cells and platelet-rich plasma. Biomaterials; 30(29):5523-5533.

8. Sawamura K, Ikeda T, Nagae M, et al (2009) Characterization of in vivo effects of platelet-rich plasma and biodegradable gelatin hydrogel microspheres on degenerated intervertebral discs. Tissue Eng Part $A$; 15(12):3719-3727.

9. Nagae M, Ikeda T, Mikami Y, et al (2007) Intervertebral disc regeneration using plateletrich plasma and biodegradable gelatin hydrogel microspheres. Tissue Eng; 13(1):147-158.

10. Tucci M, Scott V, Wingerter S, et al (2010) Evaluation of cartilage growth following sustained delivery of tgf- $\beta$ using a rat degenerated disc model: biomed 2010. Biomed Sci Instrum; 46:142-147.

\section{EDITORIAL PERSPECTIVE}

The subject of platelet-rich plasma (PRP) is a classic example of treatments gaining clinical popularity without much evidence. Treatment of one or two celebrities with alleged good outcomes will overcome even the most complete dearth of scientific studies.

Platelet-rich plasma is somewhat vaguely defined as abovenormal concentrations of platelets derived from autogenous blood through centrifugal or other physical or chemical processes - with no minimum or maximum recommended levels of platelet concentration. The potential for increased healing emerges from the observation that a number of growth factors attached to a fresh clot-as described by Gullung - may exert beneficial healing effects on surrounding tissues.

The reviewers were critical of the fact that PRP creation in this study, and in many others, is not quantified in its composition and volume. Further, the location of delivery is declared only vaguely as 'in and around the disc' and not verified by imaging, such as with computed tomography or discography. This leaves a more than passing chance that concentration of the substance may have varied strongly among the study animals due to leakage, dissipation, or inconsistent PRP preparation.

From a methodological perspective MRIs would preferably have been obtained before injection as well to assure absence of baseline changes.

Overall the reviewers were very complimentary of the study as it added some much needed basic science to a field that is largely devoid of properly conducted trials - either in bench or in clinical research [1].

1. Hall MP, Band PA, Meislin RJ, et al (2009) Platelet-rich plasma: current concepts and application in sports medicine. J Am Acad Orthop Surg; 17(10):602-608. 